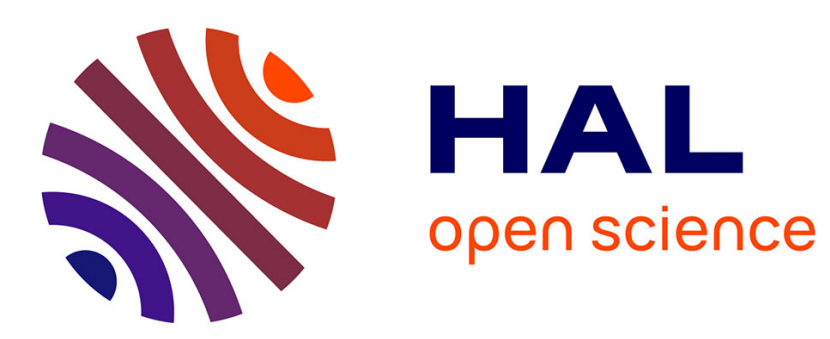

\title{
Checkpoint-based Fault-tolerance for LEACH Protocol
}

Mohamed Lehsaini, Hervé Guyennet

\section{To cite this version:}

Mohamed Lehsaini, Hervé Guyennet. Checkpoint-based Fault-tolerance for LEACH Protocol. NTMS'2014, 6th IFIP Int. Conf. on New Technologies, Mobility and Security, 2014, Dubai, United Arab Emirates. pp.1-4. hal-01230771

\section{HAL Id: hal-01230771 https://hal.science/hal-01230771}

Submitted on 19 Nov 2015

HAL is a multi-disciplinary open access archive for the deposit and dissemination of scientific research documents, whether they are published or not. The documents may come from teaching and research institutions in France or abroad, or from public or private research centers.
L'archive ouverte pluridisciplinaire HAL, est destinée au dépôt et à la diffusion de documents scientifiques de niveau recherche, publiés ou non, émanant des établissements d'enseignement et de recherche français ou étrangers, des laboratoires publics ou privés. 


\section{Checkpoint-based Fault-tolerance for LEACH Protocol}

\author{
Mohamed LEHSAINI \\ STIC Laboratory, Faculty of Technology \\ University of Tlemcen \\ Tlemcen, ALGERIA \\ m_lehsaini@mail.univ-tlemcen.dz
}

\author{
Herve GUYENNET \\ LIFC Laboratory, Department of Computer Science \\ University of Franche-Comte \\ Besancon, FRANCE \\ herve.guyennet@femto-st.fr
}

\begin{abstract}
Most routing protocols designed for wireless sensor networks provide good results in ideal environments. However, their performance degrades dramatically when nodes stop working for various causes such as loss of energy, crushed by animal or climatic conditions. In this paper, we highlight the weaknesses of LEACH (Low Energy Adaptive Clustering Hierarchy) protocol by evaluating its performance. Then we propose an improved version of this protocol based on checkpoint approach that allows it to become a fault-tolerant protocol. Finally, several simulations were conducted to illustrate the benefits of our contribution.
\end{abstract}

Keywords-LEACH; Checkpoint approach; Realistic environment; Fault-tolerance; WSN.

\section{INTRODUCTION}

Wireless sensor networks (WSN) consist of a large number of low-cost and low-powered devices that can sense, compute, and communicate with other devices for the purpose of gathering local information to make global decision about a physical environment [1]. Sensors can be deployed over a geographical area for monitoring physical phenomena like temperature, humidity, vibrations, seismic events, early fire detection, density of air pollutants and so on $[2,3]$.

WSN should have a long lifetime to accomplish the application requirements. However, In addition to resource constraints in WSN, the failure of sensor nodes is almost unavoidable due to energy depletion since they have been usually deployed in hostile environments and their batteries cannot be recharged or replaced, hardware failure, communication link errors, and so on $[3,4,5]$. Therefore, in some WSN applications, fault-tolerance has become as important issue than other performance metrics such as energy efficiency, latency and accuracy.

Generally, the consequence of these failures is that a node becomes unreachable, violates certain conditions that are essential for providing a service or returns false readings that could cause a disaster especially in critical applications. Furthermore, the above fault scenarios are worsened by the multihop communication nature of WSN. It often takes several hops to deliver data from a source node to a remote base station. Thus, failure of a single node or link may lead to missing reports from the entire region of WSN.
Therefore, since sensors are prone to failure, faulttolerance should be seriously considered in many sensing applications which are generally required to be fault-tolerant, where any pair of sensors is usually connected by multiple communication paths. Recently, several studies have dealt with fault-tolerance in WSN, particularly in the routing process. Moreover, these works focus on the detection and recovery of failures in WSN.

In this paper we propose to evaluate the routing protocol LEACH in a realistic environment in which sensor nodes can fail and links may be lost. Then, we propose an improved version of LEACH called FT-LEACH (Fault-Tolerant LEACH). In this proposed version, we use checkpoint approach to tolerate failures so that LEACH becomes a faulttolerant protocol. Furthermore, in the FT-LEACH, clusterheads aggregate data to reduce extra costs by sending only one copy of sensed data to the sink.

FT-LEACH could tolerate failures of links and therefore guarantee routing reliability in WSN while dissipating less extra energy. To illustrate the performance of FT-LEACH we conducted several simulations and we compared obtained results with those of LEACH [6] in a realist environment.

The rest of this paper is organized as follows: Section 2 describes the model parameters used; in Section 3, we present briefly the protocol LEACH; Section 4 is an improved version of LEACH; Section 5 illustrates performance analysis of LEACH and FT-LEACH in a realistic environment. Finally, we conclude our paper and discuss future research work in Section 6.

\section{MODEL PARAMETERS}

Before heading into the technical details of our contribution, we first give some definitions and notations that will be used in our paper later.

A wireless sensor network is represented by an undirected graph $G=(V, E)$, where $\mathrm{V}$ represents the set of sensor nodes and $E \subseteq V^{2}$ is the set of edges that gives the available communications: an edge $e=(u, v)$ belongs to $\mathrm{E}$ if and only if the node $\mathrm{u}$ is physically able to transmit messages to $\mathrm{v}$ and vice-versa. At each sensor node $u \in V$ is assigned a unique value to be used as an identifier, so that the identifier of $u$ is denoted by $\operatorname{Node}_{I d}(u)$. 
The 1-density of a node $u$ represents the ratio between the number of links in its 1-neighborhood (links between $u$ and its neighbors and links between two 1-neighbors of $\mathrm{u}$ ) and the 1degree of u $\delta_{l}(u)$, denoted by $\rho_{l}(u)$. It is expressed by the following formula:

$$
\rho_{1}(u)=\frac{|(v, w) \in E| v, w \in N_{1}[u] \mid}{\left|\delta_{1}(u)\right|}
$$

Where $N_{l}[u]$ is the 1-neighborhood set of $\mathrm{u}$ (the nodes that are located not more than two hops from the node u) and $\delta_{l}(u)$ is the 1-degree of $\mathrm{u}$ (the size of $N_{l}(u)$ ).

In this paper, we assume that the sensors are randomly distributed in a two-dimensional Euclidean plane P. Each sensor has an omni-directional antenna which allows it to reach with a single transmission all nodes within its vicinity and we consider that sensors have 1-hop positional information. We also assume that each sensor has a generic weight and it is able to calculate it. This weight represents the fitness of each node to be a cluster-head.

\section{PRESENTATION OF LEACH PROTOCOL}

LEACH [6,7] is a hierarchical cluster-based routing protocol for wireless sensor networks which partitions the nodes into clusters. In each cluster a dedicated node called cluster-head $(\mathrm{CH})$ and other nodes are cluster members. $\mathrm{CH}$ is responsible for creating and manipulating a TDMA schedule and sending aggregated data from nodes to a remote base station using CDMA technique to avoid interference. Moreover, this protocol runs into several rounds and each round consists of two phases:

\section{A. Setup Phase}

During this phase, cluster formation takes place. In which, each sensor node decides independently of other nodes if it will become a $\mathrm{CH}$ or not. This decision takes into account if the node is served as a $\mathrm{CH}$ for the last time i.e. the node that has not been a $\mathrm{CH}$ for long time is more likely to elect itself as a $\mathrm{CH}$.

Once $\mathrm{CHs}$ are elected, they inform their neighborhood using an advertisement packet that they become CHs. Each non- $\mathrm{CH}$ node picks the advertisement packet with the strongest received signal strength, sends the message "Join Packet" to request its corresponding $\mathrm{CH}$ to join it.

After this process, the $\mathrm{CH}$ knows the number of member nodes and their IDs. Based on all messages received within the cluster, the $\mathrm{CH}$ creates a TDMA schedule and broadcasts it to its cluster members. Then, it picks a CSMA code randomly to avoid interference when transmitting data to the base station.

\section{B. Steady-state phase}

During this phase, sensor nodes begin to send their data collected during their allocated TDMA slot to their respective $\mathrm{CHs}$. The radio of each non- $\mathrm{CH}$ node can be turned off until the nodes allocated TDMA slot, thus energy dissipation is minimized for these nodes. When all collected data have been received, the corresponding $\mathrm{CH}$ aggregates these data and sends them to the remote base station as presented by figure 1 .

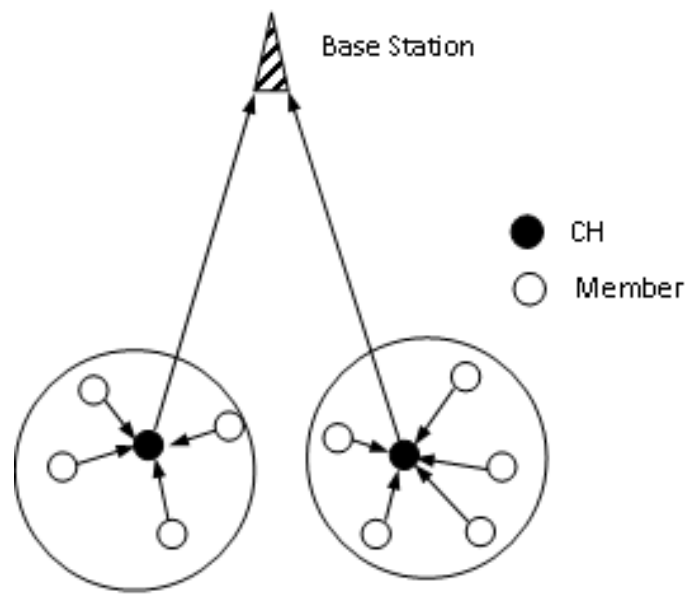

Fig. 1. Cluster formation in LEACH

LEACH is able to perform local aggregation of data in each cluster to reduce the amount of data transmitted to the base station. Although LEACH provides good results for WSN, it presents some drawbacks such as:

- $\mathrm{CH}$ selection does not take into account residual energy of nodes. Hence, it is probable that during a period there are some CHs with a small amount of energy. Thus, the latter could quickly deplete their batteries and consequently they stop working and cause holes in the target area.

- It cannot cover a large area when some sensor nodes fail.

To the best our knowledge there is no work dealing with faulttolerance in LEACH. In this context, we propose an enhanced version of LEACH called FT-LEACH so that it becomes a fault-tolerant protocol.

\section{CHECKPOINT-BASED APPROACH}

In most routing protocols, fault-tolerance has not been taken into consideration especially for LEACH protocol. In this context, we propose an enhanced version of LEACH protocol based on checkpoint approach so that LEACH becomes a fault-tolerant protocol.

The checkpoint approach is one of the most popular technique to provide fault-tolerance on unreliable and distributed systems [8]. It is a record of the snapshot of the whole network state in order to restart the application after the occurrence of some crash. The checkpoint can be stored on temporary as well as stable storage. However, the efficiency of this approach is strongly dependent on the length of the checkpointing period. Frequent checkpointing may enhance the overhead, while lazy checkpointing may lead to loss of significant computation. Hence, the decision about the size of the checkpointing interval and the checkpointing technique is a complicated task and should be based upon the knowledge about the application as well as the system [9]. Moreover, the 
checkpoint approaches present some limitations. They need stable storage to save a global consistent state periodically.

In this context, we use the base station to store availability information about cluster-heads. Each cluster-head sends periodically a message to the base station. If during a period, the base station does not receive a message from a clusterhead, it considers it as a defective node. As a result, the base station transmits a message to the cluster members concerned to elect a novel cluster-head among them. The member which has the greatest weight based on its remaining energy and its 1-density parameter as presented in Equation (2), becomes cluster-head during the remaining time of the current period.

$$
\operatorname{Weight}(s)=1 / 2 \operatorname{Energy}(s)+1 / 2 \operatorname{density}(s)
$$

\section{EVALUATION AND SIMULATION RESULTS}

In our experiments, we conducted extensive simulations to evaluate the performance of FT-LEACH and compare them with LEACH in terms respectively of the ratio of successful reception at the base station during the network's lifetime and energy consumption. To achieve these goals, the simulations have been performed in NS-2[10] using the MIT_uAMPS [11]. We have carried out these simulations with the same scenario presented in LEACH [7] in order to illustrate the performance of our contribution. Hence, we considered a network topology with 100 non-mobile sensor nodes with a sensing range of 25 meters. Sensor nodes are placed randomly in a $100 \mathrm{~m} \times 100 \mathrm{~m}$ square area by using an uniform distribution function, and the remote base station is located at position $\mathrm{x}=50, \mathrm{y}=175$, i.e. the base station was placed 75 meters outside the area where the sensor nodes were deployed. At the beginning of the simulation, all the sensor nodes had an equal amount of energy i.e. the sensor nodes started with 2 Joules of energy. Simulation parameters are presented in Table 1. Moreover, we note that system lifetime is defined as the time when last sensor dies in the sensor network.

The simulations were performed until all the sensors in the network consumed their energy and the average values were calculated after each round whose duration is 20 seconds. This duration represents the cluster timeout. It is used to prolong network lifetime and balance energy deviation among all its sensors. On expiry of this period, cluster-head's election process is triggered again. Moreover, we used the same energy parameters and radio model as discussed in [7], wherein energy consumption is mainly divided into two parts: receiving and transmitting messages. The transmission energy consumption requires additional energy to amplify the signal according to the distance from the destination. Thus, to transmit a k-bit message to a distance $\mathrm{d}$, the radio expends energy $\left(\mathrm{E}_{\mathrm{Tx}}\right)$ as described by the formula (3), where $\varepsilon_{\text {elec }}$ is the energy consumed for radio electronics, $\varepsilon_{\text {friss-amp }}$ and $\varepsilon_{\text {two-ray-amp }}$ for an amplifier. The reception energy consumption is $\mathrm{E}_{\mathrm{Rx}}=$ $\varepsilon_{\text {elec }} \times \mathrm{k}$.

$$
E_{T x}= \begin{cases}\varepsilon_{\text {elec }} * k+\varepsilon_{\text {friss-amp }} * k * d^{2} & \text { if } d<d_{\text {Crossover }} \\ \varepsilon_{\text {elec }} * k+\varepsilon_{\text {two-ray-amp }} * k * d^{4} & \text { if } d \geq d_{\text {Crossover }}\end{cases}
$$

Simulated model parameters are set as shown in Table 2 . The data size were 500 bytes/message plus a header of 25 bytes. The message size to be transmitted was:

$$
k=(500 \text { bytes }+25 \text { bytes }) \times 8=4200 \text { bits }
$$

Table 1. Model Parameters

\begin{tabular}{|l|c|}
\hline \multicolumn{1}{|c|}{ Parameter } & Value \\
\hline Network grid & $(0,0) \times(100,100)$ \\
\hline Base Station & $(50,175)$ \\
\hline$\varepsilon_{\text {elec }}$ & $50 \mathrm{~nJ} / \mathrm{bit}$ \\
\hline$\varepsilon_{\text {friss-amp }}$ & $10 \mathrm{pJ} / \mathrm{bit}$ \\
\hline$\varepsilon_{\text {two-ray-amp }}$ & $0.0013 \mathrm{pJ} / \mathrm{bit}$ \\
\hline $\mathrm{d}_{\text {Crossover }}$ & $87 \mathrm{~m}$ \\
\hline Data packet size & $500 \mathrm{bytes}$ \\
\hline Packet Header size & $25 \mathrm{bytes}$ \\
\hline Intial energy per node & $2 \mathrm{~J}$ \\
\hline Number of nodes $(\mathrm{N})$ & 100 \\
\hline
\end{tabular}

Fig. 3 shows that the ratio of successfully received packets to the base station is higher than in LEACH because in LEACH, if a cluster-head stops working information will not be forwarded to the base station while in our contribution if the cluster-head is down the cluster-head election process would be triggered and the elected cluster-head transmits the information to the base station.

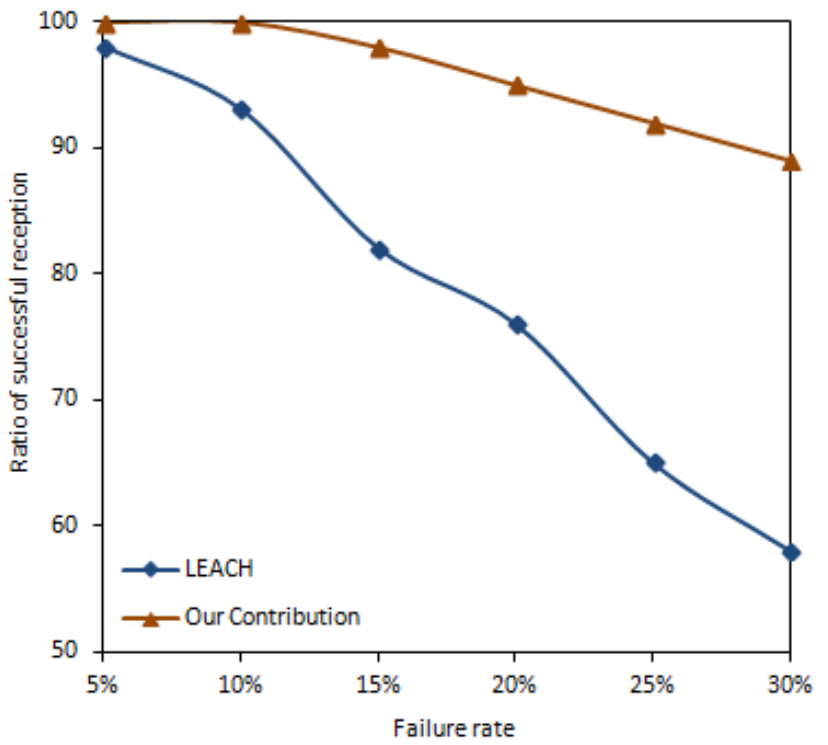

Fig. 2. Ratio of successful reception with different failure rates

Fig.4 illustrates that in our contribution, the amount of packets received at the base station during network lifetime is higher than in LEACH because in LEACH, if a cluster-head stops working information will not be forwarded to the base station and hence the energy will be lost without sending information. Furthermore, in our contribution if the cluster- 
head is down the cluster-head election process would be triggered and the elected sensor node transmits the information to the base station and hence the energy consumed reflects a transmission.

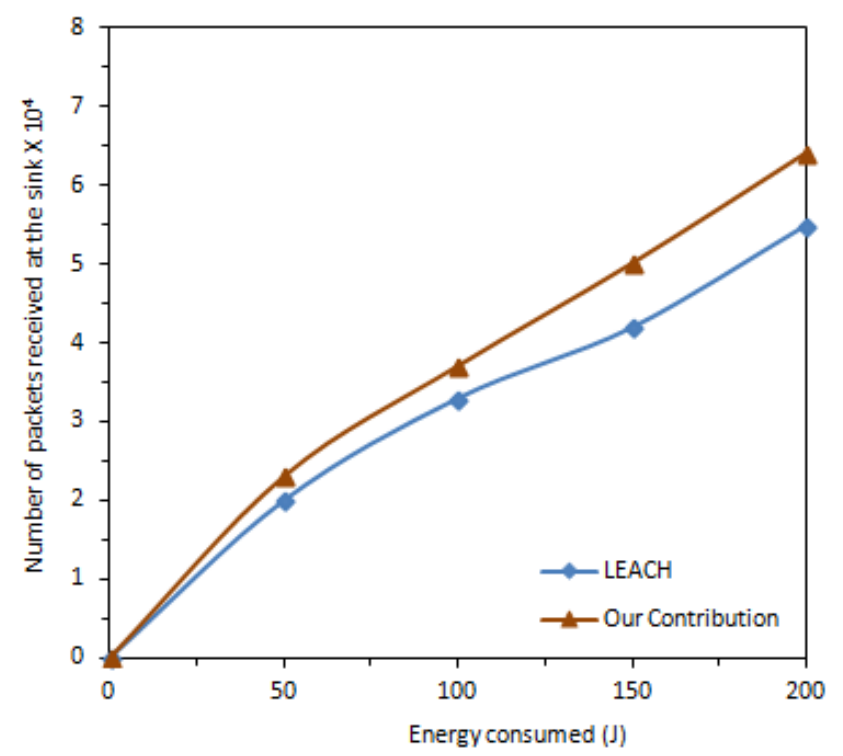

Fig. 3. Amount of packets received at the sink during network lifetime

\section{CONCLUSION}

The evaluation of LEACH in a realistic environment showed that LEACH loses performance when some nodes fail. In this context, we have proposed an enhanced version of LEACH so it becomes a fault-tolerant protocol.

Simulation results showed that our contribution has improved the performance of LEACH in terms of number of packets successfully received at the base station and energy consumption.

\section{Reference}

[1] S. Olariu and Q. Xu "Information Assurance in Wireless sensor networks", Parallel and Distributed Processing Symposium. $19^{\text {th }}$ IEEE International, pp. 236-240, Colorado USA, April 2005.

[2] G. Haosong and Y. Younghwan, "Distributed Bottleneck Node Detection in Wireless Sensor Networks", IEEE $10^{\text {th }}$ International Conference on Computer Information and Technology (CIT'2010), pp. 218-224, July 2010.

[3] A. Woo, T. Tong, and D. Culler,"Taming the underlying challenges of reliable multihop routing in sensor networks", Proceedings of the $1^{\text {st }}$ International Conference on Embedded Networked Sensor Systems (ACM), pp.14-27, Los Angeles USA, November 2003.

[4] L. Paradis and Q. Han, "A Survey of Fault Management in Wireless Sensor Networks", Journal of Network and Systems Management, Vol.15, No.2, pp.171-190, 2007.

[5] H.M. Ammari and S.K; Das, "Fault tolerance measures for large-scale wireless sensor networks", Journal ACM Transactions on Autonomous and Adaptive Systems (TAAS), Vol. 4, No. 1, pp.1-28, 2009.

[6] Heinzelman and al. "Energy-efficient communication protocol for wireless microsensor networks", IEEE Proceedings of 33rd Annual Hawaii International Conference on System Sciences (HICSS '00), Vol. 2, pp.3005-3017, Maui, Hawaii, USA, January 2000.

[7] Heinzelman and al. "An application-specific protocol architecture for wireless microsensor networks", IEEE Transactions Wireless Communications, Vol. 1, No. 4, pp.660670, October 2002.

[8] R. Garg and A. K. Singh, "Fault Tolerance in Grid Computing: State of the Art and open issues", International journal of Computer Science \& Engineering Survey, Vol. 2, No. 1, February 2011.

[9] S. Gokuldev, M. Valarmathi, "A New Checkpoint Approach for Fault Tolerance in Grid", International Journal of Computer Science and Network (IJCSN), Vol. 2, No. 3, pp.102-107, June 2013.

[10] NS-2, Network Simulator, World Wide Web, www.isi.edu/nsnam/ns/ns-build.html MIT_uAMPS LEACH NS2 Extensions, World Wide Web.

[11] www.ece.rochester.edu/research/wcng/code/index.html. 